\title{
DIVERSIDAD MORFOAGRONÓMICA DEL FRIJOL AYOCOTE EN EL ALTIPLANO CENTRO-ORIENTE DE PUEBLA
}

\section{MORPHO-AGRONOMIC DIVERSITY OF THE RUNNER BEAN IN THE CENTRAL-EASTERN HIGHLAND PLATEAU OF PUEBLA}

\author{
Luz I. López-Báez', Oswaldo R. Taboada-Gaytán, Abel Gil-Muñoz', Pedro A. López', \\ Enrique Ortiz-Torres ${ }^{1}$, Ma. Luisa P. Vargas-Vázquez ${ }^{2}$ y Rufino Díaz-Cervantes ${ }^{1}$
}

\author{
'Colegio de Postgraduados, Campus Puebla, San Pedro Cholula, Puebla, México. ${ }^{2}$ Instituto Nacional de Investigaciones Forestales, Agrícolas y \\ Pecuarias, Texcoco, Estado de México, México. \\ *Autor para correspondencia (toswaldo@colpos.mx)
}

\section{RESUMEN}

Los estudios de diversidad genética permiten conocer su magnitud e identificar las acciones necesarias para su conservación y aprovechamiento. En Phaseolus coccineus L., aun cuando se han llevado a cabo estudios de diversidad, en ningún caso se ha evaluado a las poblaciones en su área de colecta, ni se ha incluido una muestra amplia de las poblaciones locales del altiplano centro-oriente de Puebla, principal región productora de frijol ayocote en México. El objetivo de este trabajo fue determinar el nivel de variación morfoagronómica de las poblaciones locales de frijol ayocote cultivadas en la región mencionada e identificar las principales características morfoagronómicas relacionadas con la diversidad encontrada. Para ello, se evaluaron 31 poblaciones locales y cinco testigos. La evaluación se hizo en dos localidades de la zona de colecta, utilizando un diseño experimental látice con dos repeticiones por localidad. Se registraron 34 variables morfoagronómicas asociadas a características de emergencia, plántula, hoja, flor, planta, vaina, semilla y rendimiento de grano. El análisis de varianza combinado reveló que la diversidad morfoagronómica es amplia, evidenciando que la variación puede presentarse en todas las estructuras de la planta, pero principalmente en variables de precocidad, flor, vaina y semilla. Los análisis de conglomerados y componentes principales permitieron identificar cuatro grupos de poblaciones, diferenciados básicamente por precocidad, color de semilla y flor y de manera complementaria por el vigor y altura de planta, longitud del foliolo central y del peciolo, forma de semilla y peso de 100 semillas. Se concluye que la diversidad morfoagronómica presente en las poblaciones locales de frijol ayocote es amplia y que las variables días a floración, color de la flor y color de semilla fueron las más importantes para explicar la diversidad existente.

Palabras clave: Caracterización in situ, diversidad fenotípica, poblaciones locales, recursos fitogenéticos.

\section{SUMMARY}

The studies of genetic diversity enable its quantification and the identification of actions needed for its conservation and use. In Phaseolus coccineus L., even though several diversity studies have been conducted, in none of them the accessions have been evaluated in the area where they were collected, nor have included a large sample of local populations from the central-eastern highland plateau of Puebla, the main production area in México. The objective of this work was to determine the level of morpho-agronomic variation present in the local populations of runner bean cultivated in the aforementioned region, and to identify the main morpho-agronomic traits related to the found diversity. A total of 31 local populations were evaluated as well as five controls. The evaluation was conducted at two sites within the area of collection, using a lattice experimental design with two replications per site. Data were recorded on 34 morpho-agronomic traits, dealing with attributes of emergence, seedling, leaf, flower, plant, pod, seed and grain yield. The combined analysis of variance revealed that the morpho-agronomic diversity is large, showing that the variation is present in all the structures of the plant, but mostly in variables measured on flower, pod and seed. The cluster and principal components analyses allowed the identification of four groups of populations, differentiated basically by earliness, seed and flower color, and complemented by vigor and height of plant, length of central leaflet and petiole, seed shape and weight of 100 seeds. It is concluded that the morpho-agronomic diversity present in the local populations of runner bean is large, and that days to flowering, flower color and seed color, are the most important traits to explain the existing diversity.

Index words: In situ characterization, phenotypic diversity, local populations, plant genetic resources.

\section{INTRODUCCIÓN}

El frijol cultivado de la especie Phaseolus coccineus $L$. conocido en México como ayocote, patol o patola, se cultiva en regiones templado húmedas o templado semiáridas, entre los 1300 y 2650 metros de altitud, en los estados de Aguascalientes, Chiapas, Guanajuato, Hidalgo, Estado de México, Michoacán, Oaxaca, Puebla, Querétaro, Tlaxcala, Veracruz y San Luis Potosí (Cárdenas-Ramos et al., 1996); también se ha encontrado cultivado en Chihuahua, Durango y Jalisco (Lépiz, 2018; Com. Pers.) '?

De acuerdo con el SIAP (2017), durante los años 20132016, el estado de Puebla produjo en promedio $2256 \mathrm{t}$ anuales en 2590 ha, 250 ha de riego y el resto de temporal. En la entidad, los dos Distritos de Desarrollo Rural (DDR) donde se concentra el cultivo de ayocote son el DDR 04 de

'Lépiz-Ildefonso, R. (2018) Profesor Investigador, Universidad de Guadalajara 
Libres y el DDR 07 de Tecamachalco, con el 6.6 y $93.3 \%$ de la superficie sembrada y el 5.7 y $94.3 \%$ de la producción estatal, respectivamente (SIAP, 2017).

La diversidad genética se define como las variaciones heredables que ocurren en cada organismo, entre los individuos de una población y entre las poblaciones dentro de una especie. Es el resultado de las diferencias genéticas entre individuos y puede manifestarse en variaciones en secuencias de ADN, en características bioquímicas, en propiedades fisiológicas o en caracteres morfológicos (Rao y Hodgkin, 2002). Como tal, la diversidad genética es la base de la evolución, sobrevivencia y adaptación, así como de la producción agrícola; es un requisito para que los fitomejoradores puedan desarrollar nuevas variedades (Ulukan, 2011). Para medir y estudiar la variabilidad genética se puede recurrir al empleo de descriptores morfológicos, citogenéticos, bioquímicos y moleculares (Mondini et al., 2009).

Entre los trabajos orientados a estudiar la diversidad genética del frijol ayocote, a través de atributos morfológicos y agronómicos, figura el de Palmero et al. (2011), quienes caracterizaron 17 poblaciones en España mediante 32 variables y concluyeron que existe una variación significativa en características de semilla y flor. Por su parte Santalla et al. (2004), al estudiar 31 accesiones españolas, detectaron variación en caracteres cualitativos, tales como color de la flor e hipocótilo y en rasgos agronómicos relacionados con caracteres de semilla. Incluso, a nivel de una sola población se ha reportado la presencia de cierto nivel de variación fenotípica (Zeven et al., 1993).

En México, la diversidad del frijol ayocote se ha estudiado con base en datos climáticos, caracteres fenológicos y tamaño del grano. Vargas et al. (2014), en su trabajo con materiales del Eje Neovolcánico y la Sierra Madre Oriental, encontraron que el clima en el área de domesticación del frijol ayocote del centro de México actúa como una fuente importante de generación de diversidad fenológica y tamaño de semilla y que las poblaciones podían agruparse en cuatro grandes grupos con base en su origen geográfico, diferenciados también por precocidad y tamaño de semilla. La importancia de la duración del ciclo biológico y atributos de semilla y vaina también fue reportada por Vargas-Vázquez et al. (2011) para poblaciones del Carso Huasteco. Castillo et al. (2006), en su estudio de ayocotes del oriente del estado de México, encontraron que la variación morfológica fue mayor en tamaño de la semilla; con base en días a floración, número de semillas por vaina y tamaño de semilla definieron la existencia de dos grupos bien diferenciados.
Lo anterior evidencia que en el caso de México, aun cuando se han llevado a cabo estudios sobre la diversidad morfoagronómica de frijol ayocote, estos se han conducido fuera de los lugares de origen de las colectas y no se han orientado a precisar el nivel de variación fenotípica presente en las poblaciones locales cultivadas en la principal región productora del estado de Puebla. Con base en ello se realizó la presente investigación, cuyos objetivos fueron determinar la variación morfoagronómica de las poblaciones locales de frijol ayocote cultivadas en el centro-oriente del estado de Puebla e identificar las principales características morfoagronómicas que expliquen la diversidad encontrada. El conocimiento generado ampliará la información existente en torno a la variabilidad fenotípica del frijol ayocote y será de utilidad para definir estrategias de rescate, conservación y aprovechamiento de esta especie a nivel local y regional.

\section{MATERIALES Y MÉTODOS}

\section{Área de estudio}

La investigación se desarrolló en dos municipios ubicados en el centro-oriente del estado de Puebla: Chalchicomula de Sesma (DDR 04) y General Felipe Ángeles (DDR 07). El primero se localiza entre los $18^{\circ} 52^{\prime}$ y $19^{\circ} 05^{\prime} \mathrm{LN}$ y los $97^{\circ}$ $16^{\prime}$ y $97^{\circ} 34^{\prime} \mathrm{LO}$, su altitud varía entre 2380 y 5600 metros sobre el nivel del mar (msnm), predominan los suelos de tipo litosol y regosol y prevalece el clima templado subhúmedo con lluvias en verano con una temperatura y precipitación media anual de $14.6^{\circ} \mathrm{C}$ y $712 \mathrm{~mm}$, respectivamente. El segundo municipio se ubica entre los $18^{\circ} 58^{\prime}$ y $19^{\circ} 05^{\prime}$ LN y los $97^{\circ} 36^{\prime}$ y $97^{\circ} 44^{\prime}$ LO, su altitud varía entre 2160 y 2800 msnm, predominan los suelos calcisol, leptosol y regosol; el clima es templado subhúmedo con Iluvias en verano y presenta una temperatura y precipitación media anual de $16.3^{\circ} \mathrm{C}$ y $641 \mathrm{~mm}$ (INEGI, 2009).

\section{Material genético}

Se evaluaron 31 poblaciones locales de frijol ayocote cultivado con diferente coloración de grano, las cuales constituyen un grupo de materiales preseleccionados por comportamiento agronómico a partir de la evaluación de un grupo de 92 poblaciones colectadas en diferentes localidades de los municipios anteriormente mencionados. Las evaluaciones previas se llevaron a cabo durante 2014 y 2015 en tres ambientes diferentes (Ciudad Serdán, Mpio. de Chalchicomula de Sesma; San Antonio Portezuelo, Mpio. de Gral. Felipe Ángeles; Calpan, Mpio. de San Andrés Calpan), tomando como variables importantes para la selección el rendimiento de grano, color de grano y precocidad, procurando mantener la variación encontrada 
en estas características (Taboada, 2015; Com. Pers.)2. Adicionalmente se incluyeron cinco variedades testigo (Cuadro 1).

\section{Establecimiento de experimentos y manejo agronómico}

Los experimentos se sembraron en Ciudad Serdán, municipio de Chalchicomula de Sesma ( $18^{\circ} 59^{\prime} 18^{\prime \prime}$ LN y $97^{\circ}$ 26' 48" LO y 2520 msnm) y San Antonio Portezuelo, municipio de General Felipe Ángeles (180 59' 49" LN y $97^{\circ} 42^{\prime}$ 58" LO y 2170 msnm) (INEGI, 2009), los días 18 de mayo y 16 de junio de 2016, respectivamente. Los experimentos se condujeron bajo condiciones de temporal, en unicultivo, dando el manejo agronómico acostumbrado por los agricultores de las regiones de estudio y utilizando la fórmula de fertilización 60-60-00 de NPK aplicada en la primera labor. La cosecha de los experimentos se realizó los días 7 y 15 de diciembre del mismo año, respectivamente.

\section{Diseño, parcela experimental y variables evaluadas}

Se utilizó un diseño experimental de látice simple $6 \times 6$ con dos repeticiones por localidad. La parcela experimental estuvo compuesta por cuatro surcos de $5 \mathrm{~m}$ de largo y $80 \mathrm{~cm}$ de ancho, de los cuales se tomaron como parcela útil los dos centrales. Se sembraron tres semillas por mata (sitio de siembra) con una separación entre matas de $40 \mathrm{~cm}$. Después de la emergencia se aclaró a dos plantas por mata. Se registraron 34 variables, entre morfológicas y agronómicas (Cuadro 2). En la mayoría de los casos se tomó como referencia el manual de descriptores para Phaseolus coccineus L., en el que se detallan las escalas de medición utilizadas (IBPGR et al., 1983).

\section{Análisis estadístico}

Se realizó un análisis de varianza combinado para identificar las variables con significancia estadística $(p \leq$ $0.05)$ entre poblaciones, entre localidades y para la interacción población $\times$ localidad. Con base en la matriz de correlaciones se descartaron aquellas variables altamente correlacionadas $(r>0.7)$ y se seleccionaron las 10 más informativas, mismas que se sometieron a análisis multivariado. Para cada variable se calcularon los promedios por población a través de ambientes, con los que se realizó un análisis de componentes principales y se calculó la matriz de distancias euclidianas para hacer un análisis de conglomerados, aplicando el método de agrupamiento de mínima varianza dentro de grupos de Ward. Posteriormente se condujo un análisis multivariado de varianza para precisar si existían diferencias estadísticas entre los grupos formados y una prueba de medias para los grupos definidos.

${ }^{2}$ Taboada G.O.R., (2015) Profesor Investigador Asociado. Colegio de Postgraduados Campus Puebla.
Todos los análisis se llevaron a cabo utilizando el paquete estadístico SAS (SAS Institute Inc., 2013).

\section{RESULTADOS}

El análisis de varianza combinado (Cuadro 3) reveló diferencias estadísticas significativas entre poblaciones en el $56.7 \%$ de las variables evaluadas. Entre localidades hubo diferencias significativas en el $67.5 \%$ de las variables, en tanto que para la interacción "población × localidad" sólo hubo significancia estadística en tres variables: días a fructificación, densidad de semilla y rendimiento de grano por hectárea. En esta última variable se observaron diferencias significativas $(p \leq 0.01)$ tanto para poblaciones y localidades como para la interacción de ambas fuentes de variación. Se encontró que el 62 \% de las variables con diferencias estadísticas entre poblaciones se relacionaron con características de flor, vaina y semilla, mientras que el $38 \%$ restante lo estuvo con caracteres de emergencia, plántula, planta, hoja y rendimiento de grano. Los resultados anteriores muestran que existe variación fenotípica entre el conjunto de poblaciones estudiadas, siendo más notable en atributos de flor, vaina y semilla. Un aspecto adicional a resaltar es que la mayor parte de las variables no interaccionaron de manera significativa con el ambiente.

En el dendrograma obtenido, a una distancia de corte de 0.10 unidades, se identificaron cuatro grupos (Figura 1). Los estadísticos de prueba de Lambda de Wilks, Traza de Pillai, Traza de Hotelling-Lawley y Raíz máxima de Roy del análisis de varianza multivariado, mostraron que los cuatro grupos son estadísticamente distintos $(p \leq 0.01)$ reiterando el alto grado de variabilidad existente entre las poblaciones de estudio. Los datos promedio de cada variable (Cuadro 4) fueron la base principal para hacer la descripción de los grupos.

El grupo I incluyó sólo una población testigo (P99AHt), proveniente de Huamantla en el estado de Tlaxcala, de grano amarillo. Esta población se caracterizó por presentar los valores más altos en diversas variables en comparación con los demás grupos (Cuadro 4): mostró plántulas vigorosas, mayor altura de planta, foliolos más largos, peciolos de mayor longitud y ciclo tardío (86 días a floración). Además, los botones florales fueron, numéricamente, los de mayor tamaño (aun cuando todos se clasificaron como grandes), flor de color rojo, vainas más anchas, semillas más planas y el mayor peso de 100 semillas. El comportamiento de esta población, en términos de ser de ciclo tardío y presentar órganos vegetativos y estructuras reproductivas de mayor tamaño, puede atribuirse a la falta de adaptación a las condiciones edafoclimáticas de los sitios de evaluación, debido a que su origen es una región diferente a la zona de estudio. 
Cuadro 1. Datos de color de grano y sitios de colecta de las poblaciones locales de frijol ayocote evaluadas.

\begin{tabular}{|c|c|c|c|c|c|c|}
\hline Código & Color de Grano & Municipio & Localidad & Latitud & Longitud & Altitud (m) \\
\hline P3NC & Negro & $\mathrm{CHS}$ & El Veladero & $18^{\circ} 57^{\prime} 41^{\prime \prime}$ & $97^{\circ} 29^{\prime} 19^{\prime \prime}$ & 2493 \\
\hline P5BC & Blanco & $\mathrm{CHS}$ & El Veladero & $18^{\circ} 57^{\prime} 45^{\prime \prime}$ & $97^{\circ} 29^{\prime} 12^{\prime \prime}$ & 2490 \\
\hline P7MC & Morado & $\mathrm{CHS}$ & El Veladero & $18^{\circ} 58^{\prime} 02^{\prime \prime}$ & $97^{\circ} 28^{\prime} 57^{\prime \prime}$ & 2502 \\
\hline P12PC & Pinto & $\mathrm{CHS}$ & El Veladero & $18^{\circ} 57^{\prime} 42^{\prime \prime}$ & $97^{\circ} 29^{\prime} 15^{\prime \prime}$ & 2499 \\
\hline P15PC & Pinto & $\mathrm{CHS}$ & El Veladero & $18^{\circ} 57^{\prime} 58^{\prime \prime}$ & $97^{\circ} 29^{\prime} 02^{\prime \prime}$ & 2496 \\
\hline $\mathrm{P} 22 \mathrm{NC}$ & Negro & $\mathrm{CHS}$ & Las Palmas & $18^{\circ} 5545^{\prime \prime}$ & $97^{\circ} 30^{\prime} 36^{\prime \prime}$ & 2430 \\
\hline P23MC & Morado & $\mathrm{CHS}$ & San Antonio Tecajetes & $18^{\circ} 57^{\prime} 24^{\prime \prime}$ & $97^{\circ} 29^{\prime} 12^{\prime \prime}$ & 2498 \\
\hline P24BC & Blanco & $\mathrm{CHS}$ & San Antonio Tecajetes & $18^{\circ} 57^{\prime} 24^{\prime \prime}$ & $97^{\circ} 29^{\prime} 12^{\prime \prime}$ & 2498 \\
\hline P30BC & Blanco & $\mathrm{CHS}$ & San Antonio Tecajetes & $18^{\circ} 57^{\prime} 25^{\prime \prime}$ & $97^{\circ} 29^{\prime} 14^{\prime \prime}$ & 2400 \\
\hline P32BC & Blanco & $\mathrm{CHS}$ & San Antonio Tecajetes & $18^{\circ} 57^{\prime} 30^{\prime \prime}$ & $97^{\circ} 29^{\prime} 17^{\prime \prime}$ & 2501 \\
\hline P35MC & Morado & $\mathrm{CHS}$ & San Antonio Tecajetes & $18^{\circ} 57^{\prime} 26^{\prime \prime}$ & $97^{\circ} 29^{\prime} 14^{\prime \prime}$ & 2500 \\
\hline P39NC & Negro & $\mathrm{CHS}$ & San Pedro Temamatla & $18^{\circ} 55^{\prime} 29^{\prime \prime}$ & $97^{\circ} 30^{\prime} 44^{\prime \prime}$ & 2427 \\
\hline P42PC & Pinto & $\mathrm{CHS}$ & San Pedro Temamatla & $18^{\circ} 55^{\prime} 29^{\prime \prime}$ & $97^{\circ} 30^{\prime} 44^{\prime \prime}$ & 2427 \\
\hline P46BC & Blanco & $\mathrm{CHS}$ & Santa María Techachalco & $18^{\circ} 57^{\prime} 11^{\prime \prime}$ & $97^{\circ} 28^{\prime} 06^{\prime \prime}$ & 2553 \\
\hline P47AF & Amarillo & GFA & San Antonio Portezuelo & $18^{\circ} 59^{\prime} 45^{\prime \prime}$ & $97^{\circ} 43^{\prime} 14^{\prime \prime}$ & 2169 \\
\hline P50NF & Negro & GFA & San Antonio Portezuelo & $18^{\circ} 59^{\prime} 46^{\prime \prime}$ & $97^{\circ} 43^{\prime} 03^{\prime \prime}$ & 2170 \\
\hline P54NF & Negro & GFA & San Antonio Portezuelo & $19^{\circ} 00^{\prime} 06^{\prime \prime}$ & $97^{\circ} 42^{\prime} 45^{\prime \prime}$ & 2186 \\
\hline P56AF & Amarillo & GFA & San Antonio Portezuelo & $18^{\circ} 59^{\prime} 58^{\prime \prime}$ & $97^{\circ} 42^{\prime} 48^{\prime \prime}$ & 2179 \\
\hline P59NF & Negro & GFA & San Antonio Portezuelo & $18^{\circ} 59^{\prime} 45^{\prime \prime}$ & $97^{\circ} 43^{\prime} 13^{\prime \prime}$ & 2182 \\
\hline P60NF & Negro & GFA & San Antonio Portezuelo & $18^{\circ} 59^{\prime} 49^{\prime \prime}$ & $97^{\circ} 42^{\prime} 46^{\prime \prime}$ & 2168 \\
\hline P61AF & Amarillo & GFA & San Antonio Portezuelo & $18^{\circ} 59^{\prime} 49^{\prime \prime}$ & $97^{\circ} 47^{\prime} 46^{\prime \prime}$ & 2168 \\
\hline P65AF & Amarillo & GFA & San Pablo de las Tunas & $18^{\circ} 59^{\prime} 31^{\prime \prime}$ & $97^{\circ} 42^{\prime} 09^{\prime \prime}$ & 2185 \\
\hline P67BF & Blanco & GFA & San Pablo de las Tunas & $18^{\circ} 59^{\prime} 33^{\prime \prime}$ & $97^{\circ} 42^{\prime} 08^{\prime \prime}$ & 2179 \\
\hline P68BF & Blanco & GFA & San Pablo de las Tunas & $18^{\circ} 59^{\prime} 27^{\prime \prime}$ & $97^{\circ} 42^{\prime} 16^{\prime \prime}$ & 2172 \\
\hline P71BF & Blanco & GFA & San Pablo de las Tunas & $18^{\circ} 59^{\prime} 28^{\prime \prime}$ & $97^{\circ} 42^{\prime} 16^{\prime \prime}$ & 2184 \\
\hline P72AF & Amarillo & GFA & San Pablo de las Tunas & $18^{\circ} 59^{\prime} 28^{\prime \prime}$ & $97^{\circ} 42^{\prime} 16^{\prime \prime}$ & 2184 \\
\hline P73BF & Blanco & GFA & Santa Úrsula Chiconquiac & $19^{\circ} 01^{\prime} 03^{\prime \prime}$ & $97^{\circ} 40^{\prime} 59^{\prime \prime}$ & 2221 \\
\hline P84BF & Blanco & GFA & Santa Úrsula Chiconquiac & $19^{\circ} 00^{\prime} 36^{\prime \prime}$ & $97^{\circ} 40^{\prime} 49^{\prime \prime}$ & 2228 \\
\hline P85NF & Negro & GFA & Santa Úrsula Chiconquiac & $19^{\circ} 00^{\prime} 36^{\prime \prime}$ & $97^{\circ} 40^{\prime} 49^{\prime \prime}$ & 2228 \\
\hline P87NF & Negro & GFA & Santa Úrsula Chiconquiac & $19^{\circ} 00^{\prime} 44^{\prime \prime}$ & $97^{\circ} 40^{\prime} 33^{\prime \prime}$ & 2244 \\
\hline P89NF & Negro & GFA & Santa Úrsula Chiconquiac & $19^{\circ} 00^{\prime} 35^{\prime}$ & $97^{\circ} 40^{\prime} 31^{\prime \prime}$ & 2239 \\
\hline P94MHt & Morado & HTZ & Huejotzingo $(T)$ & & & \\
\hline $\mathrm{P95NHt}$ & Negro & HTZ & Huejotzingo $(T)$ & & & \\
\hline P97PSt & Pinto & SMT & San Matías Tlalancaleca ( $T$ ) & & & \\
\hline P99AHt & Amarillo & HMT & Huamantla $(T)$ & & & \\
\hline P100BIt & Blanco & TEX & CEVAMEX-INIFAP (T) & & & \\
\hline
\end{tabular}

CHS: Chalchicomula de Sesma; GFA: Gral. Felipe Ángeles; HTZ: Huejotzingo; SMT: San Matías Tlalancaleca; HMT: Huamantla; TEX:Texcoco, Edo. de Méx.; T: Testigo.

El grupo II se integró con siete poblaciones, cuatro poblaciones locales y tres testigos. De las cuatro poblaciones locales una fue de grano morado y otra de grano pinto, ambas procedentes de Chalchicomula de Sesma (CHS) y dos más fueron de grano blanco, colectadas en el municipio de General Felipe Ángeles (GFA). Los testigos presentaron coloración de grano negro $(\mathrm{P} 95 \mathrm{NHt})$, morado $(\mathrm{P} 94 \mathrm{MHt})$ y blanco (P100Blt). Los atributos distintivos de este grupo fueron plantas de menor altura, precoces (57 días a floración) y de botones florales más pequeños. En cuanto al resto de las características, las poblaciones de este grupo presentaron plántulas de vigor intermedio, foliolo central 
Cuadro 2. Variables morfológicas y agronómicas registradas en la evaluación de poblaciones locales de frijol ayocote del centro-oriente de Puebla.

\begin{tabular}{|c|c|c|c|c|}
\hline Categoría & Variable & Abreviatura & U. de medida & Muestra \\
\hline \multirow[t]{2}{*}{ Emergencia } & Número de plantas emergidas & NPLEM & Número & $\mathrm{PU}$ \\
\hline & Color de hipocótilo & COLHI & Nominal & PU \\
\hline Plántula & Vigor de la plántula & VIGPL & Nominal & PU \\
\hline \multirow[t]{5}{*}{ Hoja } & Longitud del foliolo central & LONGHO & $\mathrm{cm}$ & 5 plantas \\
\hline & Forma del foliolo central & FORHOJ & Adimensional & 5 plantas \\
\hline & Color de la hoja & COLHOJ & Nominal & PU \\
\hline & Longitud del peciolo† & LOPECIOL & $\mathrm{cm}$ & 5 plantas \\
\hline & Área foliart & ARFOLI & $\mathrm{cm}^{2}$ & 5 plantas \\
\hline \multirow[t]{5}{*}{ Flor } & Tamaño del botón floral & TAMBF & $\mathrm{mm}$ & 5 plantas \\
\hline & Color del cáliz & COLCAL & Nominal & 5 plantas \\
\hline & Días a floración & DAFLM & Días & PU \\
\hline & Apertura de la flor & APEFLO & Nominal & 5 plantas \\
\hline & Color de la flor & COLFLO & Nominal & PU \\
\hline \multirow[t]{2}{*}{ Planta } & Altura de la plantat & ALP & $\mathrm{cm}$ & 5 plantas \\
\hline & Tipo de crecimiento & TIPCRE & Nominal & 5 plantas \\
\hline \multirow[t]{7}{*}{ Vaina } & Días a fructificación & DFRUM & Días & PU \\
\hline & Número de vainas normalest & NVN & Número & 5 plantas \\
\hline & Número de vainas vanast & NVV & Número & 5 plantas \\
\hline & Curvatura de la vaina & CURVAI & Nominal & 20 vainas \\
\hline & Longitud de la vaina & LONVAI & $\mathrm{cm}$ & 20 vainas \\
\hline & Ancho de la vaina & ANCVAI & $\mathrm{mm}$ & 20 vainas \\
\hline & Longitud del pedicelo† & LONPED & $\mathrm{cm}$ & 20 vainas \\
\hline \multirow[t]{10}{*}{ Semilla } & Número de semillas normales & NSN & Número & 20 vainas \\
\hline & Número de semillas vanas & NSV & Número & 20 vainas \\
\hline & Longitud de semilla & LONSEM & $\mathrm{mm}$ & 20 semillas \\
\hline & Ancho de semilla & ANCHSE & $\mathrm{mm}$ & 20 semillas \\
\hline & Grosor de semilla & GROSEM & $\mathrm{mm}$ & 20 semillas \\
\hline & Forma de la semilla Longitud/Anchura & FORSEMA & Adimensional & 20 semillas \\
\hline & Forma de la semilla longitud/grosor & FORSEMG & Adimensional & 20 semillas \\
\hline & Peso de 100 semillas & PE100S & $g$ & 100 semillas \\
\hline & Volumen de 100 semillas & VOLSEM & $\mathrm{mm}^{3}$ & 100 semillas \\
\hline & Densidad de semillat & DENSEM & $\mathrm{g} / \mathrm{mm}^{3}$ & 100 semillas \\
\hline \multirow{2}{*}{ Rendimiento (Grano) } & Factor de desgranet & FCDESGR & Adimensional & 5 plantas \\
\hline & Rendimiento de grano por hectárea & $\mathrm{RHA}$ & $\mathrm{kg} \mathrm{ha}^{-1}$ & $\mathrm{PU}$ \\
\hline
\end{tabular}

PU: Parcela útil; †: Variables no incluidas en el manual de descriptores (IBPGR et al., 1983).

de longitud corta-intermedia y peciolos cortos. Un $57 \%$ de los materiales presentó flores color rojo y 43 \% flores blancas, el ancho de vaina fue de $13.61 \mathrm{~mm}$, tuvieron semilla plana y peso de 100 semillas de $82.53 \mathrm{~g}$.

El grupo III se formó con diez poblaciones locales, siete de ellas de CHS con tres distintas coloraciones de grano (blanco, morado y negro) y tres de GFA con grano de color blanco. El grupo se distinguió por incluir a ocho de las diez poblaciones de grano blanco estudiadas. Este conjunto se caracterizó por presentar caracteres de tipo intermedio, como: plántulas de vigor bajo-intermedio, plantas de porte intermedio, longitud del foliolo central corta-intermedia, peciolos de longitud media, ciclo intermedio-tardío (74 días a floración media) y botones florales de $15.89 \mathrm{~mm}$. El $80 \%$ de los materiales tuvo flores blancas y $20 \%$ flores 
Cuadro 3. Cuadrados medios del análisis de varianza combinado en 36 poblaciones de frijol ayocote evaluadas en el centro-oriente del estado de Puebla.

\begin{tabular}{|c|c|c|c|c|c|c|c|c|}
\hline \multirow{3}{*}{$\begin{array}{l}\text { Variables † } \\
\text { NPLEM }\end{array}$} & \multicolumn{6}{|c|}{ Cuadrados medios } & \multirow{3}{*}{$\frac{\text { C.V. (\%) }}{16.5}$} & \multirow{3}{*}{$\frac{r^{2}}{0.86}$} \\
\hline & \multicolumn{2}{|c|}{ Población } & \multicolumn{2}{|c|}{ Localidad } & \multicolumn{2}{|c|}{ Pob×Loc. } & & \\
\hline & 324.831 & ** & 2089.792 & ** & 36.743 & ns & & \\
\hline COLHI & 1.568 & $\star *$ & 0.023 & ns & 0.258 & ns & 23.6 & 0.86 \\
\hline VIGPL & 1.617 & $\star *$ & 7.895 & $* *$ & 0.461 & ns & 15.1 & 0.80 \\
\hline LONGHO & 0.642 & * & 9.476 & $\star *$ & 0.293 & ns & 8.3 & 0.76 \\
\hline FORHOJ & 0.005 & ns & 0.091 & ** & 0.005 & ns & 5.6 & 0.68 \\
\hline COLHOJ & 1.241 & ns & 0.029 & ns & 0.885 & ns & 14.9 & 0.71 \\
\hline ARFOLI & 191.022 & ns & 2275.324 & $\star *$ & 87.366 & ns & 16.6 & 0.75 \\
\hline LOPECIOL & 3.180 & $\star *$ & 69.713 & ** & 1.303 & ns & 9.8 & 0.84 \\
\hline TAMBF & 0.815 & $* *$ & 31.985 & $\star *$ & 0.220 & ns & 3.0 & 0.87 \\
\hline COLCAL & 0.712 & $\star *$ & 0.064 & ns & 0.079 & ns & 16.4 & 0.88 \\
\hline DAFLM & 189.935 & $\star \star$ & 178.995 & * & 39.123 & ns & 8.1 & 0.87 \\
\hline APEFLO & 1.303 & ns & 9.805 & $\star *$ & 1.157 & ns & 36.1 & 0.65 \\
\hline COLFLO & 5.709 & $\star \star$ & 0.270 & ns & 0.202 & ns & 11.2 & 0.97 \\
\hline ALP & 46.482 & $\star *$ & 281.702 & $\star *$ & 8.879 & ns & 8.4 & 0.85 \\
\hline TIPCRE & 0.725 & $* *$ & 0.174 & ns & 0.241 & ns & 13.9 & 0.79 \\
\hline DFRUM & 111.089 & $* *$ & 1887.730 & $\star *$ & 36.565 & * & 5.7 & 0.89 \\
\hline NVN & 51.231 & ns & 887.745 & $\star *$ & 37.970 & ns & 25.4 & 0.75 \\
\hline NVV & 3.338 & * & 109.729 & $\star \star$ & 2.594 & ns & 39.4 & 0.86 \\
\hline CURVAI & 1.214 & ns & 7.701 & * & 1.214 & ns & 42.5 & 0.65 \\
\hline LONVAI & 0.784 & ns & 25.701 & $\star *$ & 0.800 & ns & 9.9 & 0.63 \\
\hline ANCVAI & 1.907 & $\star *$ & 3.437 & ns & 1.059 & ns & 6.6 & 0.74 \\
\hline LONPED & 0.038 & ** & 0.513 & $\star \star$ & 0.022 & ns & 7.3 & 0.82 \\
\hline NSN & 0.133 & ns & 1.453 & $\star *$ & 0.076 & ns & 9.1 & 0.73 \\
\hline NSV & 0.040 & ns & 0.446 & $\star \star$ & 0.061 & ns & 37.1 & 0.73 \\
\hline LONSEM & 4.865 & $\star \star$ & 7.176 & $\star \star$ & 0.855 & ns & 5.0 & 0.88 \\
\hline ANCHSE & 27.684 & ns & 23.585 & ns & 24.799 & ns & 42.9 & 0.65 \\
\hline GROSEM & 0.282 & ns & 3.071 & $* *$ & 0.274 & ns & 7.5 & 0.69 \\
\hline FORSEMA & 0.005 & ns & 0.077 & $* *$ & 0.003 & ns & 4.1 & 0.71 \\
\hline FORSEMG & 0.076 & $* *$ & 0.076 & ns & 0.023 & ns & 6.7 & 0.77 \\
\hline PE100S & 309.510 & $* *$ & 3179.215 & $* *$ & 134.259 & ns & 11.4 & 0.83 \\
\hline VOLSEM & 838.591 & $* *$ & 2068.627 & $\star \star$ & 186.039 & ns & 14.2 & 0.87 \\
\hline DENSEM & 0.019 & $\star \star$ & 0.00005 & ns & 0.007 & * & 5.7 & 0.83 \\
\hline FCDESG & 0.001 & ns & 0.007 & $* *$ & 0.001 & ns & 3.9 & 0.70 \\
\hline $\mathrm{RHA}$ & 0.994 & $\star \star$ & 2.551 & $\star \star$ & 1.259 & $\star \star$ & 22.0 & 0.87 \\
\hline
\end{tabular}

Grados de libertad: Poblaciones: 35; Localidades:1; Poblaciones $\times$ Localidad:143; *, **: Estadísticamente significativo a P $\leq 0.05$ y P $\leq 0.01$, respectivamente; ns: no significativo; C.V.: coeficiente de variación. t: La descripción de las variables se presenta en el Cuadro 2 de la sección de Materiales y Métodos.

rojas. En el resto de características fue similar a los grupos II y IV.

Al grupo IV lo integraron 18 poblaciones y todas, excepto un testigo, fueron poblaciones nativas. Incluyó 12 colectas de GFA con color de grano negro y amarillo y cinco más de CHS de color negro, morado y pinto. El testigo (P97PSt) presentó grano pinto. Este grupo se caracterizó por incluir prácticamente a todas las poblaciones locales de grano negro evaluadas (9 de 10) y por contener sólo materiales 


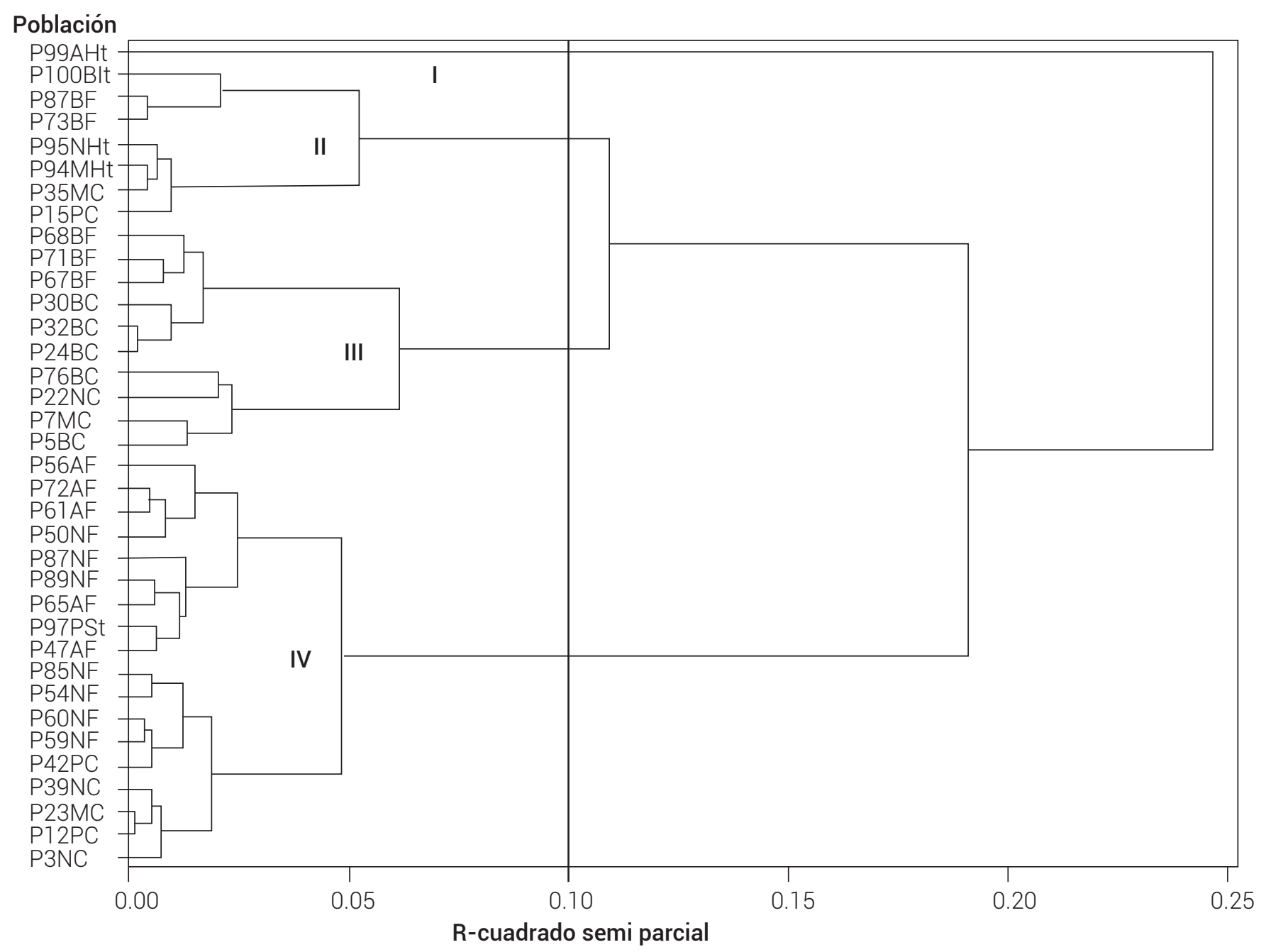

Figura 2. Dendrograma obtenido con el método de Ward para 36 poblaciones de frijol ayocote en el centro-oriente de Puebla.

Cuadro 4. Promedios para diez variables de los cuatro grupos identificados en el dendrograma de 36 poblaciones de frijol ayocote del centro-oriente de Puebla.

\begin{tabular}{|c|c|c|c|c|c|c|c|c|}
\hline \multirow{2}{*}{ Grupos } & \multicolumn{8}{|c|}{ Variables } \\
\hline & \multicolumn{2}{|r|}{ VIGPL† } & ALP & \multicolumn{2}{|c|}{ LONGHO } & \multirow{2}{*}{$\frac{\text { PECIOL }}{13.15 \mathrm{a}}$} & DAFLM & \\
\hline I & $7.00 \mathrm{a}$ & Alta & 49.43 a & $8.52 \mathrm{a}$ & Intermedia & & $86 a$ & \\
\hline ॥ & $5.14 b$ & Intermedia & $34.23 \mathrm{c}$ & $7.05 b$ & Corta-intermedia & $9.93 \mathrm{~b}$ & $57 \mathrm{c}$ & \\
\hline III & $4.42 \mathrm{~b}$ & Baja- intermedia & $37.32 \mathrm{bc}$ & $7.30 \mathrm{~b}$ & Corta-intermedia & $10.80 b$ & $74 b$ & \\
\hline \multirow[t]{2}{*}{ IV } & $5.00 \mathrm{~b}$ & Intermedia & $40.69 \mathrm{~b}$ & $7.30 \mathrm{~b}$ & Corta-intermedia & $11.03 b$ & $70 b$ & \\
\hline & \multicolumn{2}{|r|}{ TAMBF } & \multicolumn{2}{|c|}{ COLFLO } & ANCVAI & \multicolumn{2}{|c|}{ FORSEMG } & PE100S \\
\hline । & $16.59 a$ & Grande & $4.00 \mathrm{a}$ & Roja & 16.93 a & $2.97 \mathrm{a}$ & Muy plana & $121.19 a$ \\
\hline ॥ & $15.44 \mathrm{c}$ & Grande & $2.75 a b$ & Más rojas & $13.61 b$ & $2.37 b$ & Plana & $82.53 b$ \\
\hline III & $15.89 \mathrm{bc}$ & Grande & $1.93 b$ & Más blancas & $13.94 b$ & $2.35 b$ & Plana & $79.87 b$ \\
\hline IV & $16.46 a b$ & Grande & $3.97 \mathrm{a}$ & Roja & $14.08 \mathrm{~b}$ & $2.42 b$ & Plana & $89.21 \mathrm{~b}$ \\
\hline
\end{tabular}


con flores rojas. El grupo presentó también plántulas con vigor bajo-intermedio, plantas de porte intermedio, con longitud del foliolo central corta-intermedia y peciolos de $11.03 \mathrm{~cm}$. Este se clasificó como de ciclo intermedio (70 días a floración), tuvo uno de los valores más altos para tamaño de botones florales (16.46 mm), vainas de $14.08 \mathrm{~mm}$ de ancho, semilla plana y peso de 100 semillas de 89.21 g. De lo anterior se infiere que las poblaciones tendieron a agruparse principalmente con base en precocidad y tamaño de órganos, pero también por el color de grano y de la flor.

En el análisis de componentes principales se encontró que los primeros tres explicaron en conjunto el $75.2 \%$ del total de la variación observada, con contribuciones individuales de $48.4 \%$ para el primero (CP1), $15.1 \%$ para el segundo (CP2) y $11.5 \%$ para el tercero (CP3). El primer componente presentó mayor asociación con longitud del peciolo (vector propio de 0.364), peso de 100 semillas (0.363) altura de la planta (0.361), ancho de vaina (0.347), forma de la semilla dada por la relación longitud/grosor (0.331) y longitud del foliolo central (0.318); es decir, con atributos de la hoja, planta, vaina y semilla. El segundo componente estuvo asociado con la variable días a floración (0.539), tamaño del botón floral (0.450) y vigor de la plántula (-0.531). El tercer componente estuvo influido principalmente por el color de la flor (0.677). En los dos últimos componentes predominaron atributos de la flor, principalmente. Lo anterior evidencia que la diversidad morfoagronómica en ayocote se presenta en diferentes estructuras y etapas de desarrollo de la planta, tales como atributos de plántula, hoja, flor, planta, vaina, semilla y días a floración.

La Figura 3 muestra la distribución espacial de las poblaciones locales de frijol ayocote con base en los componentes principales CP1, CP2 y CP3. En la figura se identificaron los cuatro grupos definidos por el dendrograma. Se observa que el grupo I se asocia principalmente con variables del CP1, el grupo 2 con variables del CP3, el grupo 3 con variables del CP2 y el grupo 4 con variables de los CP1 y CP2. También se observa que los grupos 3 y 4 reúnen a la mayor parte de las poblaciones y que son relativamente similares entre sí; el grupo II muestra mayores diferencias entre sus integrantes y el grupo I es una población completamente diferente.

\section{DISCUSIÓN}

Los resultados mostraron una amplia diversidad morfoagronómica entre las poblaciones locales de frijol ayocote del centro-oriente del estado de Puebla, variabilidad asociada principalmente con atributos de planta, precocidad, color de flor y dimensiones de vaina y semilla. Las variables que contribuyen en mayor medida a explicar la diversidad encontrada fueron días a floración, color de la flor, ancho de vaina, peso de 100 semillas y color de semilla.

De acuerdo con Castillo et al. (2006) y López et al. (2005) actualmente gran parte de la diversidad fenotípica y genética presente en las especies cultivadas se encuentra en las poblaciones nativas conservadas por los agricultores. Con base en esta afirmación y considerando que Phaseolus coccineus es una especie predominantemente alógama (Miranda, 1974; IBPGR, 1983), se formuló la hipótesis que entre las poblaciones locales de ayocote de la región de estudio existiría una amplia diversidad morfoagronómica. Los resultados confirmaron tal supuesto, dado que para la fuente de variación "Poblaciones", la cual estuvo integrada en un $86 \%$ por poblaciones locales, se detectaron diferencias estadísticas en el $56.7 \%$ de las variables evaluadas. Al respecto, en México trabajos como los de Castillo et al. (2006) y Vargas et al. (2014), evidenciaron que entre las poblaciones nativas que integran esta especie existe un considerable nivel de variación fenotípica en el germoplasma. Tal diversidad es atribuible a la polinización prevalentemente alógama del frijol ayocote con alto grado de cruzamiento natural (Miranda, 1974; Santalla et al., 2004) y al proceso de domesticación (selección empírica) de la especie practicado por nuestros ancestros por miles de años en el área de Mesoamérica (Guerra-García et al., 2017). En este sentido, como lo señalan Mercati et al. (2015), las prácticas de selección por parte de los agricultores han influido en la estructura genética y la variabilidad de la especie.

En esta investigación, las variables que permitieron diferenciar y agrupar entre sí a las poblaciones locales bajo estudio fueron atributos relacionados con plántula, hoja, flor, vaina y semilla; los caracteres fenológicos como días a floración, altura de la planta color de la flor y color del grano fueron también particularmente importantes.

Los grupos identificados evidenciaron diferencias en el nivel de expresión de las variables previamente mencionadas y revelaron que una característica clave en el agrupamiento fue días a floración, detectándose tres niveles de precocidad. Las poblaciones locales se ubicaron en dos de ellos: precoz e intermedio. Las plantas de ciclo precoz, de menor vigor y de menores dimensiones (grupo II) posiblemente son el resultado de la selección hecha por los agricultores hacia ciclos más cortos, para enfrentar las condiciones ambientales presentes en la región de estudio (heladas tempranas y sequías). Una situación similar ha sido reportada para maíz (Zea mays L.) (Hortelano et al., 2012) en la misma región de trabajo. Esta característica de precocidad puede propiciar que la especie pueda dispersarse hacia áreas con la misma problemática, siendo 


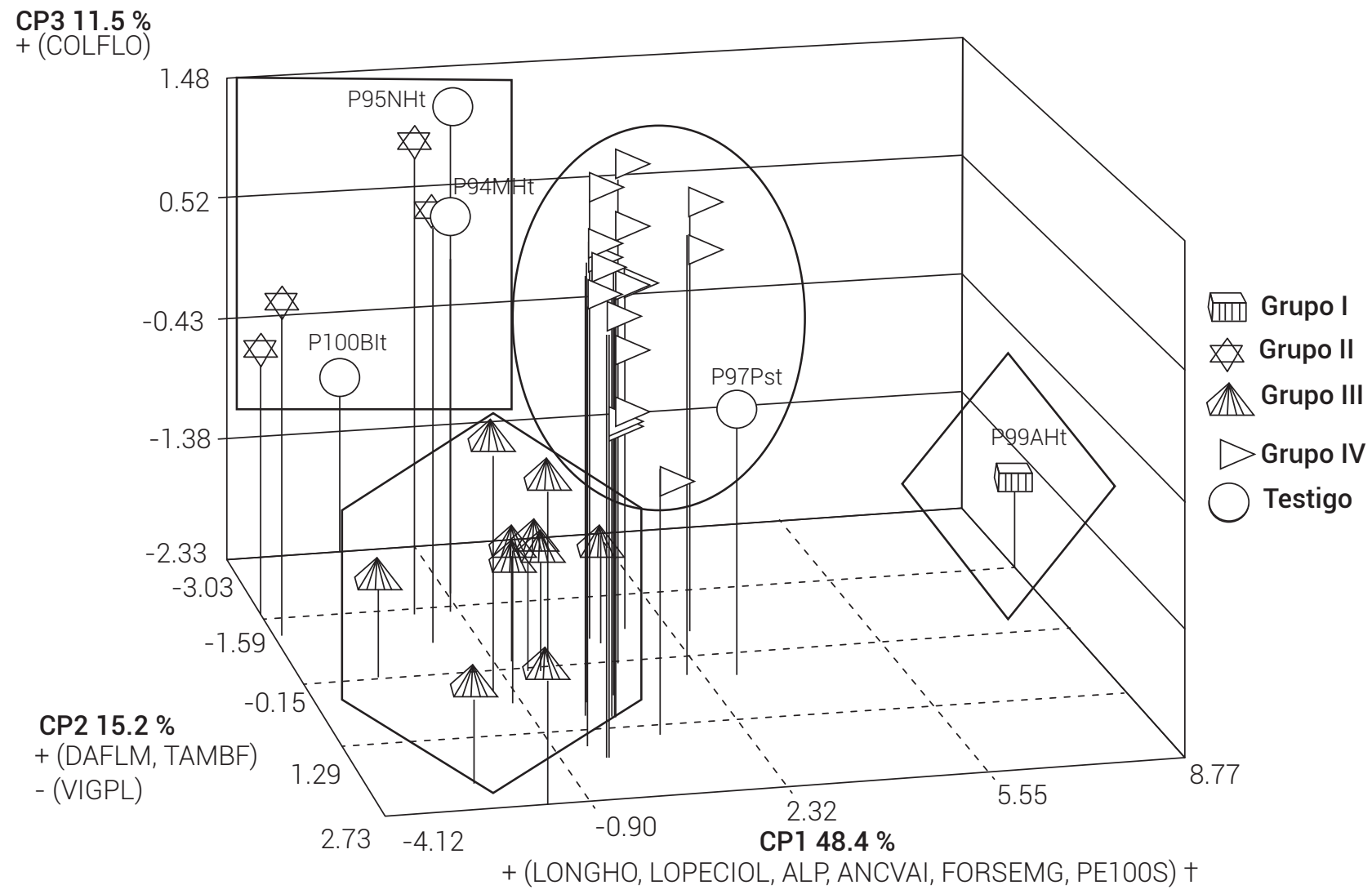

Figura 3. Dispersión de 36 poblaciones locales de frijol ayocote definida por los tres primeros componentes principales (CP) en dos municipios del centro-oriente de Puebla. + : La descripción de las variables se presenta en el Cuadro 2 de la sección de Materiales y Métodos.

así una buena fuente de germoplasma con adaptación y tolerancia a las restricciones impuestas por el ambiente (López-Romero et al., 2005; Miranda, 1974). Los grupos III y IV se clasificaron como de ciclo intermedio, aun cuando las poblaciones contenidas en el grupo III podrían considerarse como de tipo intermedio-tardío, y contuvieron a la mayor parte de las poblaciones nativas. El mayor número de poblaciones de ciclo intermedio, responde a una estrategia de los agricultores, particularmente de los de zonas de temporal, para hacer frente a las variaciones ambientales a las cuales se enfrentan, pues les da la posibilidad de aprovechar distintos períodos de crecimiento. Ello ha sido documentado ampliamente por Muñoz (2005).

En los grupos II y IV, que contuvieron poblaciones locales, se encontraron los cinco tipos de coloración de grano identificados; por su parte, el grupo III estuvo compuesto mayormente por poblaciones de grano blanco y flores blancas, en tanto que el IV incluyó prácticamente a todas las poblaciones de grano negro y flores rojas. Se ha señalado (Hernández, 1985) que en el cultivo de maíz la presencia de diferentes tipos de coloración de grano responde a la necesidad del agricultor de tener materiales adecuados para condiciones ecológicas diversas, así como para variar la dieta y atender creencias y tradiciones. Ello podría ser el caso del cultivo de ayocote en la zona de estudio.

En cuanto a la variación en el color de la flor es probable que ésta pueda ser el resultado de un criterio de selección por parte de los agricultores para distinguir entre granos pigmentados y aquellos que no lo son. De hecho, Miranda (1974) menciona que el color blanco de la flor ha sido una característica sujeta a selección. Adicionalmente, la asociación entre flor blanca y grano blanco y entre flor roja y grano pigmentado ya ha sido documentada en la literatura (Santalla et al., 2004). Lo anterior indica que entre las poblaciones locales del altiplano centro-oriente de Puebla, los caracteres de color de flor y semilla están asociados a los criterios de selección de cada agricultor. Esto puede también asociarse con el rendimiento de grano, ya que las colectas con grano negro y blanco presentaron los rendimientos promedio más altos a través de ambientes con 2843 y $2794 \mathrm{~kg} \mathrm{ha}^{-1}$, respectivamente; el rendimiento promedio de las colectas con color de grano morado, 
pinto y amarillo fue de 2437, 2378 y $2320 \mathrm{~kg} \mathrm{ha}^{-1}$, de forma respectiva. Esto evidencia el alto potencial de rendimiento de grano que poseen las variedades locales, debido a sus atributos genéticos y al proceso de selección a que han sido sometidas por parte de los agricultores y su adaptación a condiciones de clima templado semihúmedo o templado semiárido, donde el frijol común (Phaseolus vulgaris L.) tiene serios problemas de producción.

Los resultados del presente estudio están en concordancia con lo reportado por Castillo et al. (2006), quienes encontraron que los valores de días a floración sirvieron como criterio para la conformación de grupos, en tanto que en el trabajo de Palmero et al. (2011) se distinguieron claramente dos grupos principales, los cuales correspondieron a variedades de flores blancas y de flores rojas. En Phaseolus vulgaris, las variables de días a floración y hábito de crecimiento de acuerdo a su origen geográfico, permitieron clasificar a 200 poblaciones en cinco grupos (Vargas-Vázquez et al., 2008).

La variación en características de la vaina y la semilla se debe a intereses económicos y de consumo (Spataro et al., 2011), atributos que los agricultores normalmente toman en cuenta al momento de decidir qué materiales de frijol ayocote conservar. Las variables relacionadas con la semilla son rasgos importantes para los agricultores y para los potenciales compradores y consumidores. Algunos de los hallazgos reportados en este estudio son consistentes con los de otros investigadores que han estudiado la diversidad morfoagronómica del frijol ayocote. Por ejemplo, Palmero et al. (2011) reportaron diferencias estadísticas entre poblaciones, específicamente en las variables de longitud y ancho de la semilla; en este estudio, las diferencias estadísticas se presentaron en las variables longitud de semilla, forma de la semilla dada por la relación longitud/ grosor, peso de 100 semillas, volumen de 100 semillas y densidad de semilla; sin embargo, no hubo significancia estadística en el ancho de semilla, lo que refleja cierta homogeneidad en esta característica.

Por otro lado, Vargas-Vázquez et al. (2011) reportaron que los primeros tres componentes principales, constituidos por variables originales medidas en la semilla, explicaron el 80 \% del total de la variación fenotípica; las variables altamente explicativas fueron: grosor de semilla, largo de semilla y relación largo/ancho de semilla, patrón de moteado, tipo de patrón de moteado y color más oscuro de las accesiones de ayocote del Carso Huasteco. En este estudio, la forma de la semilla dada por la relación longitud/ grosor y el peso de 100 semillas resultaron mayormente explicativas en lo que respecta al conjunto de variables de semilla. Un último aspecto a resaltar es que la forma de la semilla de las poblaciones locales correspondió a la categoría "plana"; ello concuerda con la manera en la cual se conoce al frijol ayocote en el norte del estado de Morelos: yepatlaxtle, que en el vocablo náhuatl significa "frijol plano" (Monroy y Quezada-Martínez, 2010).

El presente estudio constituye el primer acercamiento al conocimiento de la diversidad fenotípica entre poblaciones locales de frijol ayocote provenientes del centro-oriente del estado de Puebla in situ. Sin embargo, se considera conveniente realizar investigaciones adicionales que permitan seguir contribuyendo al conocimiento de la diversidad de $P$. coccineus y promuevan el aprovechamiento eficiente de sus atributos potenciales en un programa de mejoramiento genético.

\section{CONCLUSIONES}

La diversidad morfoagronómica presente en las poblaciones locales de frijol ayocote del altiplano centro-oriente de Puebla es amplia y se encuentra distribuida en todas las estructuras de la planta, principalmente en atributos de planta, flor, vaina y semilla. Las características relevantes para describir la diversidad fueron número de días a floración, color de flor, color y tamaño de semilla. Otras variables como vigor y altura de planta, longitud del foliolo central y del peciolo, ancho de vaina y forma de la semilla complementan la diferenciación entre poblaciones y entre grupos.

\section{BIBLIOGRAFÍA}

Cárdenas-Ramos, F., J. S. Muruaga-Martínez y J. A. Acosta-Gallegos (1996) Catálogo banco de germoplasma de Phaseolus spp. del Instituto Nacional de Investigaciones Forestales y Agropecuarias. INIFAP. $421 \mathrm{p}$.

Castillo M. M., P. Ramírez V., F. Castillo G. y S. Miranda C. (2006) Diversidad morfológica de poblaciones nativas de frijol común y frijol ayocote del oriente del Estado de México. Revista Fitotecnia Mexicana 29:112-119, http://www.redalyc.org/articulo. oa?id:61029203.

Guerra-García A., M. Suárez-Atilano, A. Mastretta-Yanes, A. Delgado-Salinas and D. Piñeiro (2017) Domestication genomics of the open-pollinated scarlet runner bean (Phaseolus coccineus L.). Frontiers in Plant Science 8:1891, https://doi.org/10.3389/fpls.2017.01891.

Hernández X. E. (1985) Maize and man in the greater southwest. Economic Botany 39:416-430, https://doi.org/10.1007/BF02858749.

Hortelano S. R. R., A. Gil M., A. Santacruz V., H. López S., P.A López y S. Miranda C. (2012) Diversidad fenotípica de maíces nativos del altiplano centro-oriente del estado de Puebla, México. Revista Fitotecnia Mexicana 35:97-109, http://www.redalyc.org/articulo. oa?id:61023300002.

INEGI, Instituto Nacional de Estadística, Geografía e Informática (2009) Compendio de información geográfica municipal. http://www.inegi. org.mx/geo/contenidos/topografia/compendio.aspx (Agosto 2017).

IBPGR, International Board for Plant Genetic Resources (1983) Phaseolus coccineus descriptors. International Board for Plant Genetic Resources, Rome. $34 \mathrm{p}$.

López-Romero G., A. Santacruz-Varela, A. Muñoz-Orozco, F. Castillo-González, L. Córdova-Téllez y H. Vaquera-Huerta (2005) Caracterización morfológica de poblaciones nativas de maíz del Istmo de Tehuantepec, México. Interciencia 30:284-290, http://www.uacm. 
kirj.redalyc.redalyc.org/articulo.oa?id:33910407.

Mercati F., G. Catarcione, A. R. Paolacci, M. R. Abenavoli, F. Sunseri and M. Ciaffi (2015) Genetic diversity and population structure of an Italian landrace of runner bean (Phaseolus coccineus L.): inferences for its safeguard and on-farm conservation. Genetica 143:473-485, http://dx.doi.org/ 10.1007/s10709-015-9846-1.

Miranda C. S. (1974) Evolución de Phaseolus vulgaris y P. coccineus. In: E. Mark Engleman (ed.) Contribuciones al Conocimiento del frijol (Phaseolus) en México. Colegio de Postgraduados, Chapingo, México. pp: 83-98.

Mondini, L., A. Noorani and M. A. Pagnotta (2009) Assessing Plant Genetic Diversity by Molecular Tools. Diversity 19-35, https://doi. org/10.3390/d1010019.

Monroy R. y A. Quezada-Martínez (2010) Estudio etnobotánico del frijol yepatlaxtle (Phaseolus coccineus L.), en el área natural protegida Corredor Biológico Chichinautzin, Morelos, México. Avances en Investigación Agropecuaria 14:23-34, http://www.redalyc. org/articulo.oa?id:83716113002.

Muñoz 0. A. (2005) Centli Maíz. Segunda Edición. Colegio de Postgraduados. Texcoco, Estado de México. 211 p.

Palmero D., C. Iglesias, M. De Cara, J. C. Tello and F. Camacho (2011) Diversity and health traits of local landraces of runner bean (Phaseolus coccineus L.) from Spain. Journal of Food Agriculture \& Environment 9: 290-295, https://doi.org/10.1234/4.2011.1953.

Rao V. R. and T. Hodgkin (2002) Genetic diversity and conservation and utilization of plant genetic resources. Plant Cell, Tissue and Organ Culture 68:1-19, https://doi.org/10.1023/A:1013359015812.

Santalla M., A. B. Monteagudo, A. M. González and A. M. De Ron (2004) Agronomical and quality traits of runner bean germplasm and implications for breeding. Euphytica 135: 205-215, https://doi. org/10.1023/B:EUPH.0000014912.07993.e7.

SAS Institute Inc. (2013) SAS Version 9.4. Statistical Analysis System
Institute. Cary, NC, USA

SIAP, Servicio de Información Agroalimentaria y Pesquera (2017) http:// nube.siap.gob.mx/cierre_agrícola/ (Octubre 2017).

Spataro G., B. Tiranti, P. Arcaleni, E. Bellucci, G. Attene, R. Papa and V. Negri (2011) Genetic diversity and structure of a worldwide collection of Phaseolus coccineus L. Theoretical and Applied Genetics 122:1281-1291, https://doi.org/10.1007/s00122-011-1530-y.

Ulukan, H. (2011) The use of plant genetic resources and biodiversity in classical plant breeding. Acta Agriculturae Scandinavica, Section B - Soil \& Plant Science 61:97-104, https://doi. org/10.1080/09064710903573390.

Vargas V. M. L. P., J. S. Muruaga M., N. Mayek P., A. Pérez G. y E. Ramírez S. (2014) Caracterización de frijol ayocote (Phaseolus coccineus L.) del Eje Neovolcánico y la Sierra Madre Oriental. Revista Mexicana de Ciencias Agrícolas 5:191-200, http://www.redalyc. org/articulo.oa?id:263129784002

Vargas-Vázquez P., J. S. Muruaga-Martínez, S. E. Martínez-Villarreal, R. RuizSalazar, S. Hernández-Delgado, N. Mayek-Pérez (2011) Diversidad morfológica del frijol ayocote del Carso Huasteco de México. Revista Mexicana de Biodiversidad 82:767-775, http://www.redalyc.org/articulo.oa?id:61029203

Vargas-Vázquez M. L. P., J. S. Muruaga-Martínez, P. Pérez-Herrera, H. R. Gill-Langarica, G. Esquivel-Esquivel, M. Á. Martínez-Damián,... y N Mayek-Pérez (2008) Caracterización morfoagronómica de la colección núcleo de la forma cultivada de frijol común del INIFAP. Agrociencia 42:787-797, http://www.redalyc.org/articulo. oa?id:30211207005

Zeven A. C., H. H. Mohamed, J. Waninge and H. Veurink (1993) Phenotypic variation within a Hungarian landrace of runner bean (Phaseolus coccineus L.). Euphytica 68:155-166, https://doi. org/10.1007/BF00024164 
